\title{
THE HERSCHEL EFFECT AND SOLAR PHOTOGRAPHY
}

\author{
R. JAYANTHAN \\ McMath-Hulbert Observatory, The University of Michigan
}

(Received 6 November, 1969)

\begin{abstract}
A method of photographing the solar image in the UV part of the solar spectrum is described. The resulting images show the bright photospheric network of Sheeley and Chapman; these have been recorded at the small central distance of $20^{\circ}$. During a flare of importance $1 \mathrm{~b}$ near a spot group, no detectable changes in the spots, their relative positions or the bright photospheric network could be observed.
\end{abstract}

\section{Introduction}

Solar tower telescopes are generally designed as auxiliary instruments feeding spectrographs or spectroheliographs of high resolving power. Thus the design criteria for such telescopes consist mainly in providing an image of adequate brightness which also has adequate angular resolution. For direct photography of the sun, it is usually necessary to provide sufficient attenuation of the solar radiation so that reasonable exposure times on conventional low-sensitivity emulsions result. Refractors of small aperture with enlarging lens systems are employed and in addition optical filters are used to cut down the wavelength range of the continuum. It is not surprising therefore that photographic studies of the photosphere have almost exclusively been carried out with telescopes of this type. Occasionally, suitable additional instrumentation has been developed (for example at the Mount Wilson Observatory) to make white-light photographs in tower telescopes. However, systematic studies of the solar photosphere in tower telescopes are rare. A procedure which seems to be eminently suited for direct photography in a tower telescope is described below.

\section{The Herschel Effect}

If a photographic emulsion is exposed to blue light and subsequently to red light before development, some of the effects of the original exposure are destroyed; this was discovered by Sir John Herschel in 1840 and is known as the Herschel effect. The effect has been used commercially in the production of emulsions called 'autopositive' film for making direct positive prints of line drawings. These emulsions can be easily adapted for the photography of the solar image at the prime focus of tower telescopes which have only reflectors in the optical train. Such photographs seem to hold considerable promise for solar research. The use of autopositive film for solar photography was first described by Colton (1960).

The autopositive film marketed by Eastman Kodak Company is a slow emulsion which is pre-exposed to blue light at the factory. Exposure of the emulsion to yellow light erases the effect of the original exposure and leaves the emulsion sensitive to 
only the short range of wavelengths between 3200 and $3700 \AA$, with a peak sensitivity at about $3500 \AA$. The emulsion can be handled and processed with the normal room lights on. Use of these films at the McGregor Tower Telescope of the McMathHulbert Observatory has not required modification or additional instrumentation of any kind. The autopositive emulsion is attached to the slit plate of the vacuum spectrograph with suitable clamps, exposed for about one-half second to the solar image, processed in diluted developer and fixed. Figures 1 and 2 show a large sunspot and photospheric features near the limb photographed in this way.

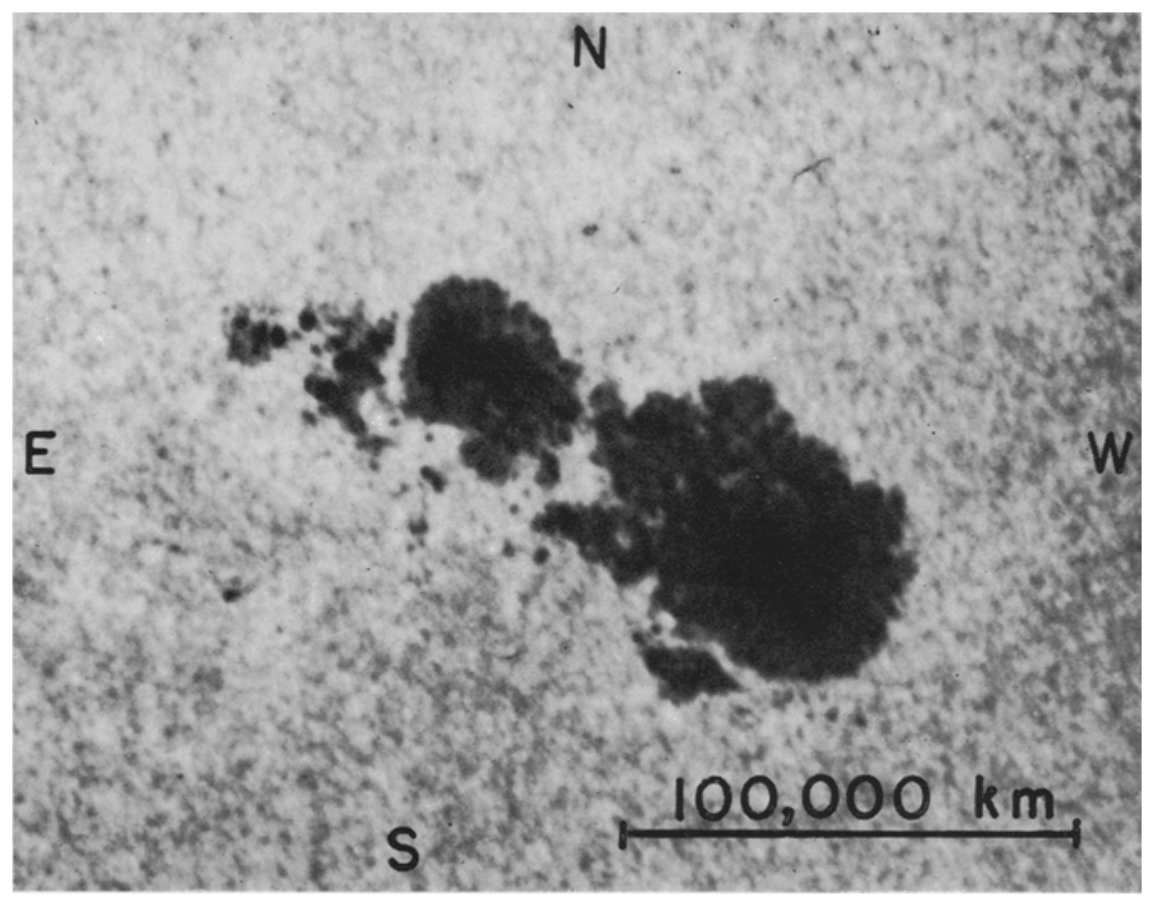

Fig. 1. A large sunspot group photographed on June 11, 1969 on a solar image of size $285 \mathrm{~mm}$.

\section{The Photospheric Network}

Sheeley (1967) observed gaps in the Fraunhofer lines and attributed them to regions in the photosphere which had magnetic fields of several hundred gauss; these fields have been called by him non-spot photospheric magnetic fields. Following this discovery, Chapman and Sheeley (1968) carried out an extensive survey with the spectroheliograph at the Kitt Peak Observatory and reported on the bright photospheric network (these appear as photospheric faculae near the limb) which is exactly coincident with the non-spot magnetic fields. The central intensities of the absorption lines in the spectra of these features are higher and the equivalent widths lower than in the normal photosphere for low-excitation lines of neutral atoms; the wings of strong 
lines also are weakened. The phenomenon thus seems to affect almost all lines, including those which are unaffected by the Zeeman effect. Therefore, by photographing the solar image even in a fairly large wavelength range of the spectrum containing many absorption lines, it should be possible to record the bright photospheric network. The procedure described above records the solar image in a region of the spectrum ( 3200 to $3700 \AA$ ) which has a great many absorption lines and the photospheric features on such images should correspond to the bright photospheric network. Faculae near the limb show up with greater contrast than on 'white-light'

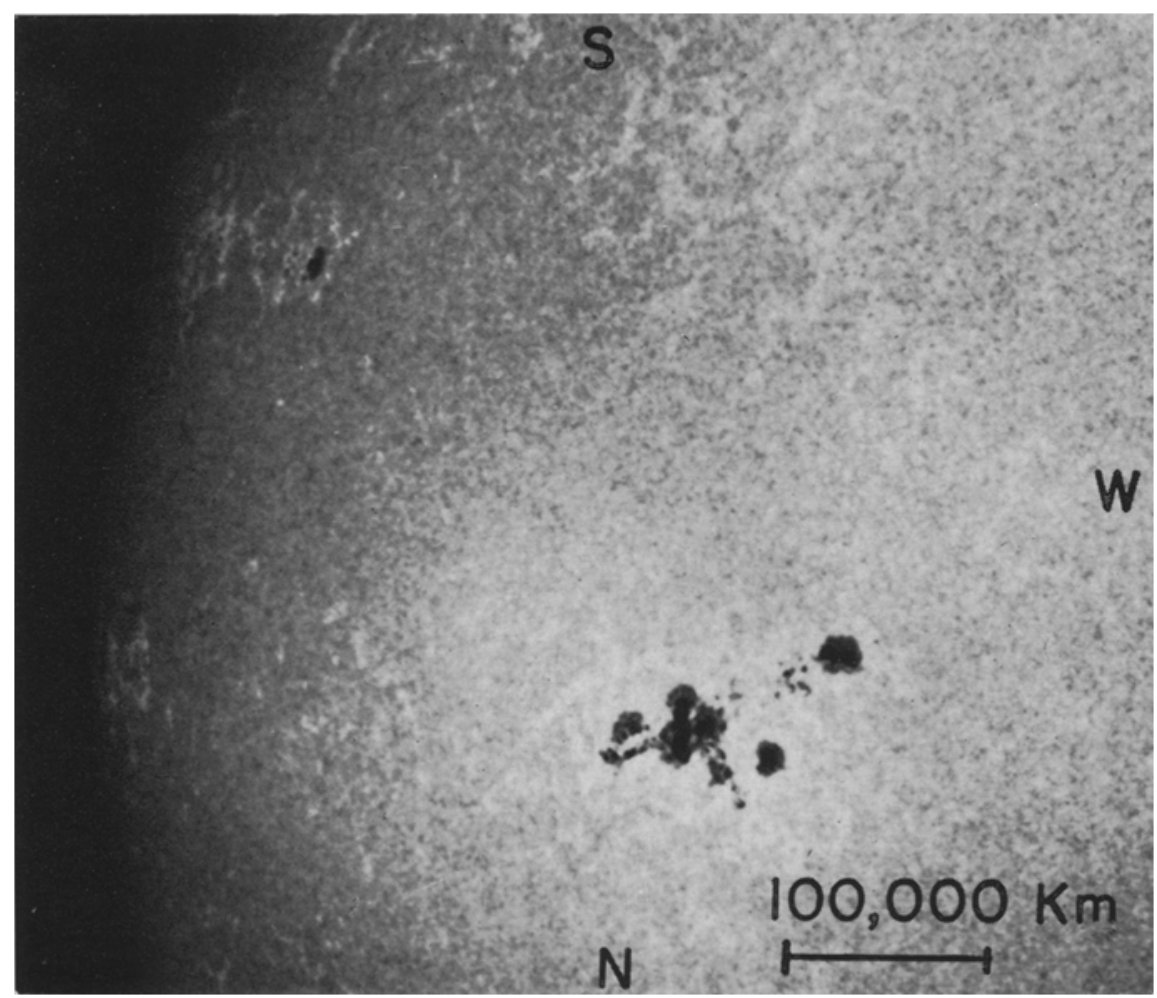

Fig. 2. A contact print of region near the east limb on August 25, 1969.

pictures made in the visible range and can be photographed even when only $20^{\circ}$ from the solar center. Experiments to reduce the pass band to increase the contrast so that the network can be photographed easily all over the disk so far have been unsuccessful.

Schmahl (1967) observed (in the continuous spectrum) sites on the solar disk where calcium plages were present, using photo-electric methods. He concluded that these features which could be observed to have increased intensities represented photospheric faculae. Since faculae could be distinguished even at the solar center, he saw no need to postulate a temperature deficit in the deeper layers. The model which 
described his observations best required the gas pressure to be less in faculae than in the undisturbed photosphere. The existence of a magnetic field in faculae ensures pressure balance with their surroundings. The present identification on direct photographs of the bright photospheric faculae as photospheric magnetic regions is in harmony with the conclusions of Schmabl.

The technique described above seems to provide a simple, convenient method of recording the magnetic field regions at the photospheric level for a large part of the solar disk. The spatial resolution is that of the tower telescope and hence high (limited only by 'seeing'); the exposures are short (a small fraction of a second) and hence an entire center of activity can be photographed at effectively the same instant of time (cf. magnetographs). Unfortunately the technique gives no way in which the direction of the magnetic vector can be determined, and information on the magnetic polarities and field strengths cannot be deduced. Nevertheless the technique should be valuable in the study of the time variations of the structures of the photospheric network and their association with other aspects of solar activity.

For example, a flare of importance $1 \mathrm{~b}$ occurred in a spot group (S17, W70) on July 12 , 1969 during the period $14^{\mathrm{h}} 23^{\mathrm{m}}$ UT to $15^{\mathrm{h}} 32^{\mathrm{m}}$ UT. The $\mathrm{H} \alpha$ flare however was not associated with accompanying energetic radiations. Pictures of the photosphere in the UV made before, during and after this flare indicate no apparent changes in the sunspots, their relative positions and the photospheric faculae.

\section{References}

Chapman, G. A. and Sheeley, N. R.: 1968, Solar Phy's. 5, 442.

Colton, H. C.: 1960, Eastman Kodak Pamphlet No. M-15.

Herschel, J. F. W.: 1840, Phil. Trans. 131, 1.

Schmahl, G.: 1967, Z. Astrophys. 66, 81.

Sheeley, N. R.: 1967, Solar Phys. 1, 171. 